\title{
Approach of Training Working Staff of Power System Operation Mode Based on State Evaluation
}

\author{
Qian Feng, Fan Youping, Liu Chen, Ai Yunfei \\ Power Dispatching and Communication Center Of Guangdong Province, Guangzhou,China \\ Email: ayf543ah@yahoo.cn
}

Received 2013

\begin{abstract}
An approach of training working staff of power system operation mode based on state evaluation is proposed. In terms of knowledge features of power system operation mode, we studied a training method based on evaluation of learning state. This training method makes individual learning for different individual condition to give them ability to grasp learning points quickly, evaluate real-time learning effect, update learning style in time and summarize problems after one learning stage, so that learners can master professional knowledge in constant summaries and feedbacks. Obvious effects can be obtained on discontinuous learning time that trainees can master basic theories associated with their working and operations adapted to practical work quickly.
\end{abstract}

Keywords: Power System Operation Mode; Professional Training; State Evaluation

\section{Introduction}

Operation mode department assumes important task in power system, constantly requiring a large number of calculation and analysis to ensure security and stability of power system operation. At present, practical training effect of operation mode is not that ideal. Such training just crammed the knowledge to learners that acceptance and mastery couldn't be reflected in time. Since base and professional background of working staff vary, the training emphasis is different for each trainee.

As the notion of scientific training becomes popular, staffs, especially knowledge staffs, pay more attention to get scientific and efficient knowledge training provided by the entity. This training state evaluation method exactly provides a good insight into improving training effect and reconstructing more scientific and more practical training system.

\section{Basic Model of Scientific Training Evaluation}

\subsection{Basic Model of Training System}

In the whole training system, training evaluation is a very critical component. Without it, the system couldn't be intact. An intact training system model is shown in Figure 1.

A scientific training system analyzes organization, work and individual to determine training demand. Then it identifies objectives, by which it can determine objects, contents, time and ways. After that, it draws out training program, which is in fact the specification and operation of training objectives. Next is to carry out training activities and final to evaluate.

\subsection{Model of Training Cyclic Process}

Training process should be a systematically cyclic process. In this cyclic system, both evaluation and training demand analysis are important parts, belonging to independent core part. When there was no training evaluation, cycle would not occur, so that training is directly from demand analysis to implementation. Figure 2 shows a simplified training cyclic system model.

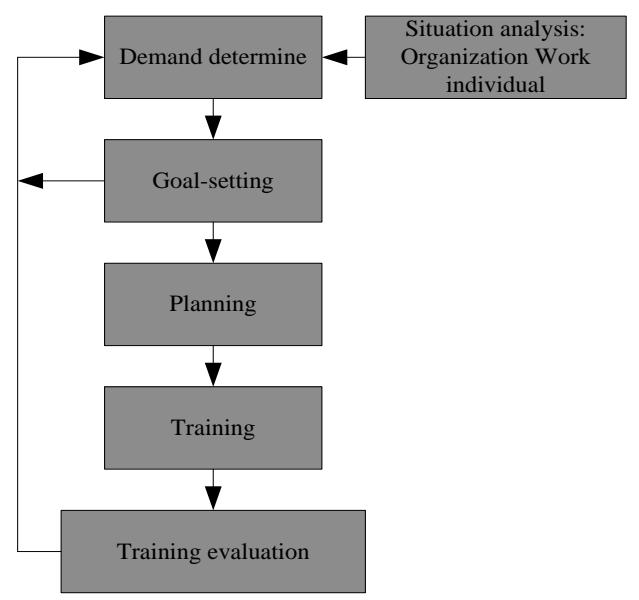

Figure 1. basic model of training system. 
Considering training evaluation as part of the cycle will contribute to the entity's feeding back practical operation results to decision-making layer by monitoring and correcting of training process. After the feedback, we compare results with predetermined objectives to find out deviations and then adjust and correct practical work to make it go on wheels on original plan or adjusted plan by a series of judgments.

\subsection{Scientific Training Evaluation Method}

D.Kirkpartrick divided training effect into four progressive levels that are reaction, learning, action and result in terms of evaluation depth and difficulty, and provided answers to four questions (see Table 1).

\subsection{Particularity and Demand of Training Working Staff of Power System Operation Mode}

Training simulators of power system at home and abroad have been developed since 1970s. it started focusing on generation units of thermal power plants, then extending to simulation training on power network and substations. Dispatcher Training Simulation (DTS) is widely used in all national dispatching centers as a powerful tool. Substation Training Simulation System (STS) needs to not only build realistic working environment and simulate potential contingencies of substation as well as perform normal operations and contingency handling training, but also be able to make full use of computer technology to implement training from various aspects.

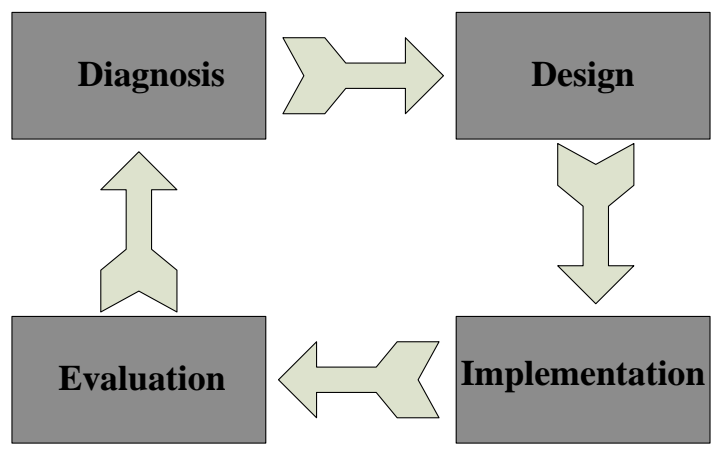

Figure 2. Simplified training cyclic system model.

Table 1. Four-level evaluation method of D.Kirkpartrick.

\begin{tabular}{|c|c|}
\hline rank & questions \\
\hline 1.reaction & $\begin{array}{l}\text { Which aspects of training program can meet trainees' } \\
\text { demand? }\end{array}$ \\
\hline 2.learning & What did trainees gain from this program? \\
\hline 3.action & Has trainees' behavior changed through training? \\
\hline 4.result & $\begin{array}{l}\text { Did behavior change have positive impact on organi- } \\
\text { zation? }\end{array}$ \\
\hline
\end{tabular}

Work of power system operation mode involves more theoretic application due to its particularity. Therefore, its training is different with conventional training. The working characteristics are mainly as following:

(1) Strong theoretical

Work of power system operation mode involves more theoretic application, such as power network structure, power flow calculation, security and stability analysis and judgment etc. For working staff of operation mode, mastering basic theoretic knowledge of power system well is the base of effective work.

(2) Many involved software operating

Power system operation mode has to analyze network structure of this province or this gird constantly, while complexity of which determines that working staff must skillfully use power system computation software such as BPA. They can make right judges quickly and submit analysis report at first time only if they mastered common computation software.

(3) Busy work

Operation mode department has to analyze future grid state in advance, and make corresponding arrangements like expansion or maintenance, so work of this department is ahead of grid operation. When there are important activities, festivals or critical maintenance and expansion, operation mode department needs to submit analysis report in advance, which means that work of this department is busy.

Thus, training for operation mode needs a systematic and individual method. In the new approach, professional points can pass to trainees visually and accurately for easy understanding; trainees can arrange time at their own wills and save learning progress to provide feedbacks for state evaluation so that improve learning efficiency.

\section{Training Method for Working Staff of Power System Operation Mode Based on State Evaluation}

\subsection{Overall Framework of Training}

Combined with scientific training system model, this paper proposed an approach of training working staff of power system operation mode based on state evaluation in terms of the working characteristics. Overall training process is shown in Figure 3. This method consists of 4 components: state analysis of training effect, that is, dividing process into three stages and setting learning state variables for each stage to analyze and test; evaluation of specific learning state, which represents by combining training software evaluation, trainee's self-evaluation and trainer's evaluation to evaluate each stage objectively; updating of training state, implemented by training software's resulting state historical database; Cumulative 
assessment of training state, in other words, to divide the training into 4stages to perform different level of evaluation, respectively, and finally make total assessment.

\subsection{Evaluation of Specific Learning State}

Evaluation of learning state is divided into three stages: state examination before learning, state evaluation during learning, state updating after learning. Distribution is as Figure 4 shows.

\section{1) State examination before training}

This stage includes two parts that are readiness exams of basic knowledge and practical skill. Since the basis varies, examining readiness of trainees can give a rough idea of their knowledge and skill base to make training pertinence.

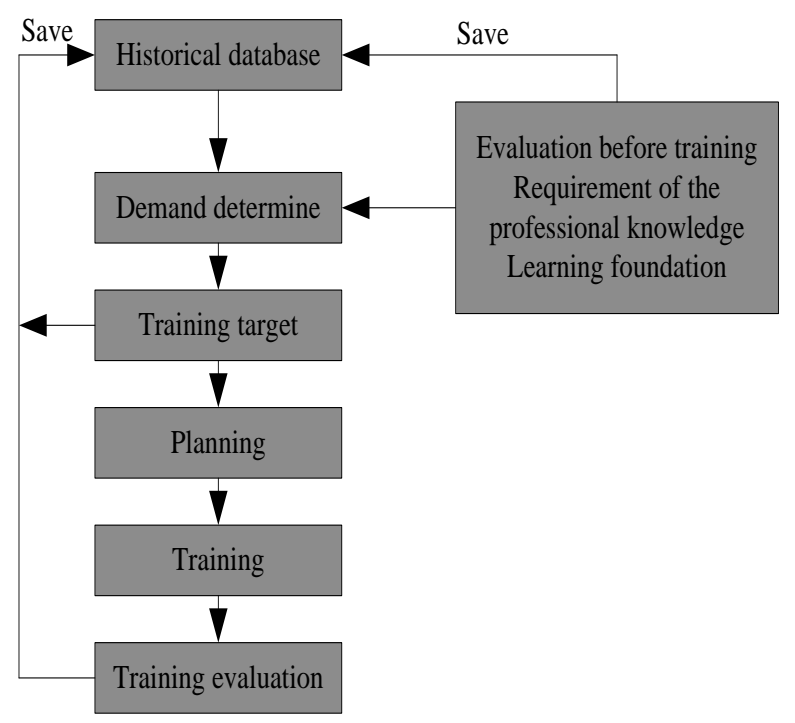

Figure 3. Process of overall training method.

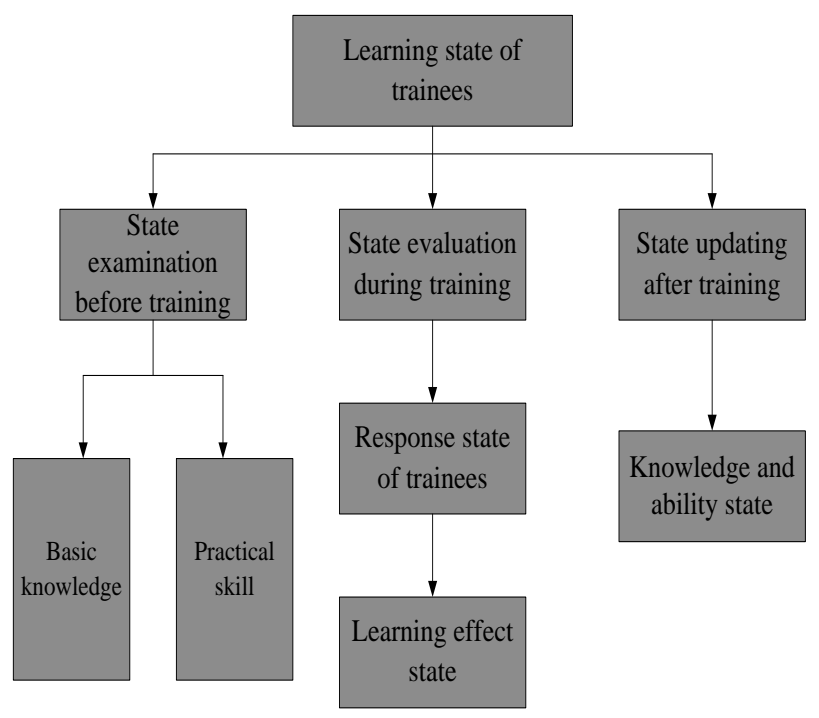

Figure 4. Distribution of learning state.
Readiness exam of basic knowledge is mainly about theoretic knowledge. Before training, we can know about knowledge readiness of trainees by a quick electronic test. Test content is knowledge points of courses to be taken, and test questions are harder as the question number increases. In the end, trainees' readiness is graded according to their answers. Mastering level of knowledge decides the amount of energy they should pay to courses to be trained. If a trainee has already gotten corresponding professional knowledge base, he can jump this part.

Readiness exam of practical skill is more complex. Because of the little time available for exam, this exam is mainly about self-test. Before training one skill, such as use of BPA and setting sheet formation of spare power automatic switching device, trainees get familiar with the software on themselves and self-evaluate their familiarity and proficiency. Self-test is done with software function blocks to let trainees judge their behavior. After test, the system will ask trainees to self-grade in terms of given levels, thus obtain the rank of skill readiness. System then provides purposeful training for each trainee by the self-test results. If someone has a good skill base and reaches a good mastering level, he can jump this part.

\section{2) State evaluation during training}

Response state and learning effect state during training. In the training, trainees' response reflects their learning effect. If they respond quickly and actively, they have a good learning state and are easy to accept knowledge; while if they respond slowly, they haven't fully mastered the knowledge, so they need to continue consolidating what they have learned. In this stage, after single knowledge point is taught, training system will pop up choice questions. Through trainees' response condition and answering time, their response state can be provided to determine learning effect. If response to one point is slow and accuracy is not high, it demonstrates that the trainee has some difficulty in receiving this point and system will repeat it again more slowly. To each test, system will record corresponding state so that can evaluate learning state after training.

\section{3) State updating after training}

Overall test of entire knowledge module is needed after training, which is a test with time limit. Simple basic knowledge has a short time limit, requiring trainees to give answers quickly at first time; difficult essay questions and calculation questions also have time limits corresponding to difficulty level, requiring trainees can master the points well and apply them and skills fast. Test result after training is measured with two variables that are total score and answering time. Although one may get a high score, if he gave answers near or on the time limit, he is demonstrated to just master for temporary and system still suggests the trainee to consolidate after training. 
After the training, the summary can be done as four-stage evaluation of D.Kirkpartrick (Table2). Incorporating specific training content, we can evaluate whether the training has prominent effect on improving trainees' knowledge and skill state.

\subsection{State Updating}

Evaluation process of learning state is shown in Figure 5. In the training, state variables of each stage for trainees reflect specific condition of entire training and are recorded and preserved for future training.

Table 2. Four-stage state evaluation after training.

\begin{tabular}{cl}
\hline rank & \multicolumn{1}{c}{ questions } \\
\hline 1.reaction & $\begin{array}{l}\text { Are trainees satisfied with knowledge points in training } \\
\text { program? }\end{array}$ \\
2.learning & $\begin{array}{l}\text { Have trainees mastered knowledge theory or operation } \\
\text { skills from training program? }\end{array}$ \\
3.action & Does behavior in working change through training? \\
4.result & $\begin{array}{l}\text { Does behavior change contribute to successful working } \\
\text { of operation mode department? }\end{array}$
\end{tabular}

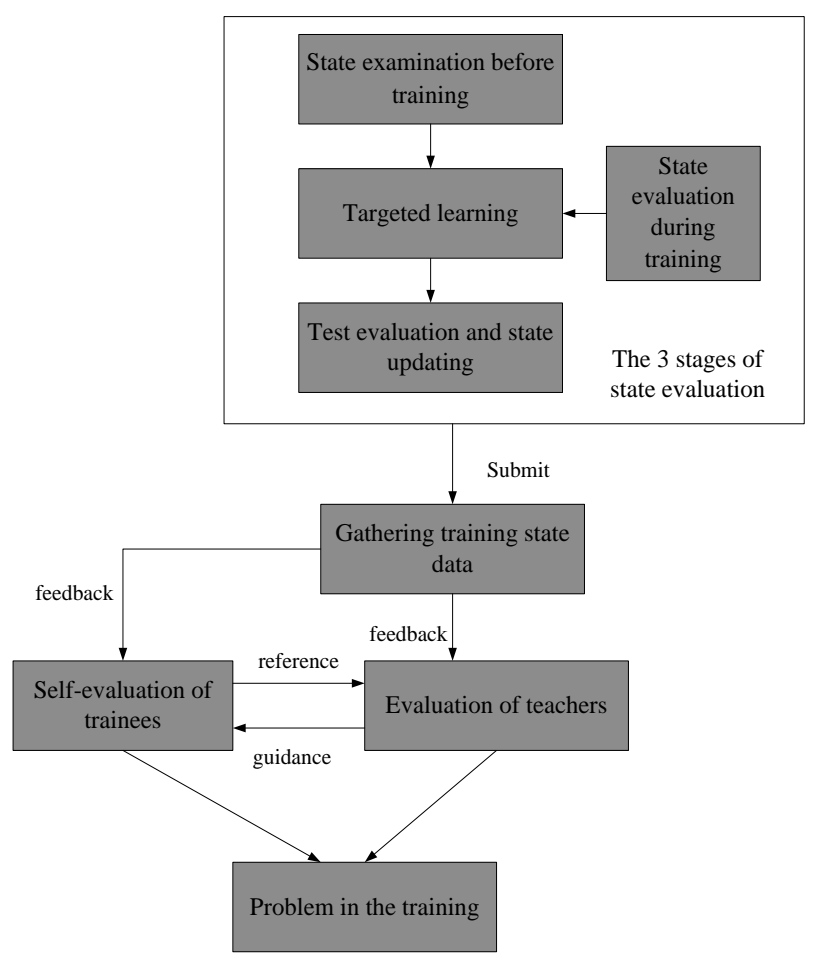

Figure 5. Evaluation process of learning state.

System files states of each training class which will accumulate to form training state historical data base. To every stage in training, we compare the state variables of the same stage (state before, during, after training) in different knowledge training to get change curve. Whether a trainee has already mastered knowledge is judged from the curve. Learning state is good with uptrend, that is, someone has well digested what we taught, while bad with violation, meaning that problem is to be found and state should be adjusted.

\subsection{State Accumulative Evaluation}

As figure 6 shows, training consists of three knowledge subsystems: basic training system, professional skills training system, as well as working process and management training system.

Basic training introduces criteria and basic knowledge associated with operation mode, stage evaluation of which is in form of electronic questions.

Skill training involves the use of BPA and so on. Working process and management is about operation mode reports of each period, analysis of power plant's switching into system, electricity guarantee scheme, generation source management and operation management of stability control equipment.

The second and third subsystems have to set tests for professional skills in addition to electronic questions. Professional skills are tested from multi-aspects. Training software simulates specific working scene for trainees to judge and respond to test their ability of knowledge application and quick response.

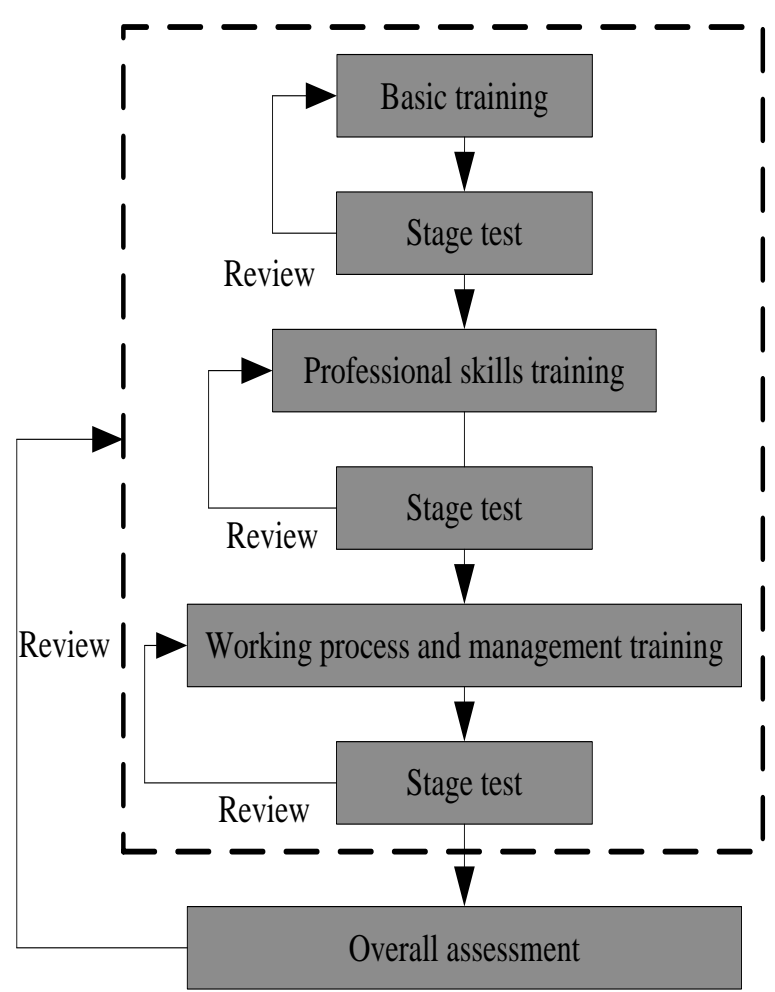

Figure 6. Stage accumulative evaluation process of training state. 
Overall assessment is done after all training of the three knowledge subsystems. Basic theoretic knowledge is permeated in comprehensive questions and applications to test trainees' ability of applying knowledge. After all training stages, trainees can go back to previous training blocks to review knowledge and consolidate what they have learned by constant feedbacks.

\section{Fuzzy Comprehensive Assessment}

Fuzzy comprehensive assessment is to quantize practical fuzzy variables using assemble and fuzzy math. This assessment can evaluate trainees during training and monitor trainers. Consider skill training of BPA calculation software. It illustrates the process of fuzzy comprehensive assessment during training.
In terms of features of BPA skill training, tasks such as data input program, power flow calculation and short circuit current calculation are designed as corresponding knowledge points.

To every point, there are three test forms. (1) completion ; (2)simulate operation test on graphical interface; (3error correction. Through three tests above, we can exam mastering condition of trainees to provide basis for next decision-making.

Specific procedure of comprehensive assessment is:

(1) During test, if a trainee answers one question correctly, the corresponding cognitive ability or abilities would be 1 ; if wrongly, be 0 . Which terms the question effects is given by experts. After the trainee finishes all questions associated with one point, he can obtain a test record table as table 3 shows.

Table 3. knowledge test record table.

\begin{tabular}{|c|c|c|c|c|c|c|}
\hline \multicolumn{7}{|c|}{ Reflections of each cognitive ability in training } \\
\hline number & memorization & comprehension & application & analyzation & integration & evaluation \\
\hline 1 & 1 & 0 & 1 & 1 & 1 & 0 \\
\hline 2 & 1 & 1 & 1 & 0 & 1 & 1 \\
\hline 3 & 1 & 0 & 0 & 1 & 1 & 1 \\
\hline$\ldots$ & $\ldots$ & $\cdots$ & $\ldots$ & $\ldots$ & .. & $\ldots \ldots$ \\
\hline $\mathrm{n}$ & $\mathrm{n}$ & $\mathrm{n}$ & $\mathrm{n}$ & $\mathrm{n}$ & $\mathrm{n}$ & $\mathrm{n}$ \\
\hline
\end{tabular}

(2) After test, representation of each terms obtained from trainee to some type of test can be provided.

$$
Z_{f}=\left(z_{1}, z_{2}, z_{3}, z_{4}, z_{5}, z_{6}\right)
$$

where, $z_{i} \in[0,1], 0 \leq i \leq 6, z_{i}$ indicates value of one cognitive ability.

$$
z_{i}=\frac{R_{i j}(1)}{n}
$$

where, $0 \leq \mathrm{j} \leq \mathrm{n}$ ( $\mathrm{n}$ is the number of test questions). $R_{i j}(1)$ is the times of correctly answering ith cognitive ability in this test. Cognitive ability includes six terms of memorization, comprehension, application, analyzation, integration, evaluation.

(3) Test pattern of each knowledge element involves the three forms previously described. So, evaluation matrix of mastering degree to each test can be constituted after test.

$$
A=\left[\begin{array}{llll}
z_{11} & z_{12} & \cdots & Z_{16} \\
Z_{21} & Z_{22} & \cdots & Z_{26} \\
Z_{31} & Z_{32} & \cdots & Z_{36}
\end{array}\right]
$$

Where, the first row represents the six evaluation values of mastering degree in completion. The second row represents that in simulated operation. The third row represents that in error correction.

(4) Weights of three forms are determined by

$$
T=\left(T_{1}, T_{2}, T_{3}\right), T_{1}+T_{2}+T_{3}=1
$$

$\mathrm{T} 1$ is the weight of completion; T2 is the weight of simulated operation; T3 is the weight of error correction.

$$
T_{i}=t_{i} /\left(t_{1}+t_{2}+t_{3}\right)
$$

Where, $t_{1}, t_{2}, t_{3}$ are the average answering evaluation value of one test form after several tests, respectively. They are pre-set by experts at the beginning use of system and can be adjusted correspondingly with training effect after some time of training test.

(5) Calculate final mastering level after test. Evaluation results are:

$$
\begin{aligned}
B & =T \square A=\left(T_{1}, T_{2}, T_{3}\right)\left[\begin{array}{cccc}
z_{11} & z_{12} & \cdots & z_{16} \\
Z_{21} & z_{22} & \cdots & z_{26} \\
Z_{31} & z_{32} & \cdots & z_{36}
\end{array}\right] \\
& =\left(B_{1}, B_{2}, B_{3}, B_{4}, B_{5}, B_{6}\right)
\end{aligned}
$$

(6) Calculate trainee's mastering condition $\mathrm{M}$ of this knowledge point:

$$
M=\sum_{i=1}^{6} B_{i} \square Q_{i}
$$

Where, $Q_{\mathrm{i}}$ is the weight of mastering ability of one knowledge point.

\section{Conclusions}

Using scientific training system model, we developed an 
approach of training working staff of power system operation mode based on state evaluation, effectively overcoming problems of busy work as well as non-systematic and concentrated training. Fuzzy comprehensive evaluation is applied in state evaluation, which dealt with state data of test after training and reflects training state in time to improve learning efficiency.

\section{REFERENCES}

[1] Donald Kirpatrick. Techniques for Evaluation Training Programs [M], Training and Development v50 p54-9 Ja'96.

[2] Laurie J.Bassi, George Benson, and Scott Cheney. The Top Ten Trends [M],Training and Development(Alexandria,Va.) v50 p38-54 N’96.

[3] ZHANG Yue. Fuzzy mathematics method and its application [M]. China Coal Industry Publishing House, 1996.
[4] TIAN Ling. Research on Fuzzy Comprehensive Evaluation of Electric Energy Purchasing Scheme Based on Analytic Hierarchy Process [J]. Power System Technology, 2005,29(7):23-27.

[5] QI Hong,YUAN Wei. Research on Distance Learner's Learning Characters and Strategies [J]. Distance Education in China, 2005, (17): 34-41.

[6] LI Haiyan. Distance Learners' Behaviors at Different Stages of the Learning Process [J]. Distance Education in China, 2009, (3):44-47.

[7] GAO Bo. The Research of Application of Meta-cognition and Fuzzy Theory in Electric Power System Simulating Training [D]. North China Electric Power University, 2007.

[8] CAO Ronghui, DONG Hongwei. Remote Education Training System for Power System Based on IP [J]. Northeast Electric Power Technology, 2006,27(12): 31-3 